\title{
On the Dependence of the Relativistic Angular Momentum of a Uniform Ball on the Radius and Angular Velocity of Rotation
}

\author{
Sergey G. Fedosin \\ PO box 614088, Sviazeva str. 22-79, Perm, Perm Krai, Russia \\ fedosin@hotmail.com
}

Keywords: relativistic angular momentum; moment of inertia; neutron star.

\begin{abstract}
In the framework of the special theory of relativity, elementary formulas are used to derive the formula for determining the relativistic angular momentum of a rotating ideal uniform ball. The moment of inertia of such a ball turns out to be a nonlinear function of the angular velocity of rotation. Application of this formula to the neutron star PSR J1614-2230 shows that due to relativistic corrections the angular momentum of the star increases tenfold as compared to the nonrelativistic formula. For the proton and neutron star PSR J1748-2446ad the velocities of their surface's motion are calculated, which reach the values of the order of $30 \%$ and $19 \%$ of the speed of light, respectively. Using the formula for the relativistic angular momentum of a uniform ball, it is easy to obtain the formula for the angular momentum of a thin spherical shell depending on its thickness, radius, mass density, and angular velocity of rotation. As a result, considering a spherical body consisting of a set of such shells it becomes possible to accurately determine its angular momentum as the sum of the angular momenta of all the body's shells. Two expressions are provided for the maximum possible angular momentum of the ball based on the rotation of the ball's surface at the speed of light and based on the condition of integrity of the gravitationally bound body at the balance of the gravitational and centripetal forces. Comparison with the results of the general theory of relativity shows the difference in angular momentum of the order of $25 \%$ for an extremal Kerr black hole.
\end{abstract}

\section{Introduction}

In the general theory of relativity, the angular momentum of a ball can be calculated using the metric [1], since it is necessary to take into account both the effect of gravity and centripetal acceleration, which change the metric properties of the volume of the ball. In the special theory of relativity in the slow-rotation approximation, the influence of the metric in the first approximation can be neglected.

The angular momentum of an ideal uniform ball in classical mechanics is calculated by the formula:

$$
J=\frac{8 \pi \rho_{0} \omega a^{5}}{15}=\frac{2 m \omega a^{2}}{5}
$$

where $\rho_{0}$ is the mass density of the ball's matter, $\omega$ is the angular velocity of rotation, $a$ is the ball's radius, $m$ is the ball's mass.

However, formula (1) does not take into account the relativistic effect of the momentum's dependence on the velocity for each of the ball's particles, so that (1) is applicable only at low rotation velocities. According to the special theory of relativity, in a body moving at a constant linear velocity, the mass density increases in proportion to the Lorentz factor $\gamma=\frac{1}{\sqrt{1-v^{2} / c^{2}}}$, where $v$ is the velocity of the body, $c$ is the speed of light. At the same time, the volume of the body decreases in inverse proportion to the Lorentz factor, as a consequence of the Lorentz length contraction. As a result, the body mass $m$ as the product of the mass density by the volume remains unchanged. The same applies to the mass element $d m=\rho_{0} d x d y d z$. 
In the case under consideration, the ball's mass element is moving along the rotation circumference and not along a straight line. Thus, our calculations will be limited to the accuracy, with which the special theory of relativity approximates rotation of bodies, and inertial reference frames approximate rotating non-inertial reference frames, where acceleration of rotation occurs.

We will first try to calculate the relativistic angular momentum of the ball in the Cartesian coordinates $x, y, z$. In these coordinates, the volume element of a fixed uniform ball is given by the formula $d V=d x d y d z$. Multiplying the volume element by the mass density $\rho_{0}$ we find the mass element of the ball: $d m=\rho_{0} d V$. Suppose the ball rotate at the angular velocity $\omega$ about the axis $O Z$ of the fixed coordinate system with the origin at the center of the ball.

Let some mass element be at a distance $R=\sqrt{x^{2}+y^{2}+z^{2}}$ from the ball's center and have the relativistic momentum $d \mathbf{p}=\gamma_{r} d m \mathbf{v}$, while the linear velocity of motion of the mass element $\mathbf{v}=[\boldsymbol{\omega} \times \mathbf{R}]$ is given by the vector product of the angular velocity $\boldsymbol{\omega}$ by the radius-vector $\mathbf{R}$.

Since the vector $\boldsymbol{\omega}$ is directed along the axis $O Z$, only two components of the linear velocity are not equal to zero: $\mathbf{v}=(-\omega y, \omega x, 0)$. To calculate the angular momentum of the mass element we need to multiply vectorially the radius-vector by the momentum: $d \mathbf{J}=[\mathbf{R} \times d \mathbf{p}]$. Then we need to integrate $d \mathbf{J}$ over all the ball's mass elements in order to find the total angular momentum $\mathbf{J}$. The components $d J_{x}$ and $d J_{y}$ are proportional to $x$ and $y$, accordingly, so that after integration over the entire volume of the ball, the components $J_{x}$ and $J_{y}$ will be equal to zero.

Since the vector $\mathbf{J}$ has only one non-zero component $J_{z}$ which is directed along the axis $O Z$, then taking into account the dependence of the Lorentz factor $\gamma_{r}=\frac{1}{\sqrt{1-\frac{\omega^{2}\left(x^{2}+y^{2}\right)}{c^{2}}}}$ on the coordinates, for this vector's magnitude $J=J_{z}$ we find the following:

$$
J=\int d J=\omega \int \gamma_{r}\left(x^{2}+y^{2}\right) d m=\rho_{0} \omega \int \frac{x^{2}+y^{2}}{\sqrt{1-\frac{\omega^{2}\left(x^{2}+y^{2}\right)}{c^{2}}}} d x d y d z
$$

We will substitute the limits of integration in the volume integral (2) and integrate with respect to the variable $z$ :

$$
\begin{aligned}
& J=\rho_{0} \omega \int_{-a}^{a}\left[\int_{y=-\sqrt{a^{2}-x^{2}}}^{y=\sqrt{a^{2}-x^{2}}}\left\{\int_{z=-\sqrt{a^{2}-x^{2}-y^{2}}}^{z=\sqrt{a^{2}-x^{2}-y^{2}}} \frac{x^{2}+y^{2}}{\sqrt{1-\frac{\omega^{2}\left(x^{2}+y^{2}\right)}{c^{2}}}} d z\right\} d y\right] d x= \\
& =2 \rho_{0} \omega \int_{-a}^{a}\left[\int_{y=-\sqrt{a^{2}-x^{2}}}^{y=\sqrt{a^{2}-x^{2}}} \frac{\left(x^{2}+y^{2}\right) \sqrt{a^{2}-x^{2}-y^{2}}}{\sqrt{1-\frac{\omega^{2}\left(x^{2}+y^{2}\right)}{c^{2}}}} d y\right] d x .
\end{aligned}
$$

Apparently, the integral with respect to the variable $y$ in (3) refers to elliptic integrals and does not reduce to elementary functions, which makes it difficult to calculate it. Therefore, the clearness 
is lost in predicting the dependence of the angular momentum $J$ on the angular velocity, mass or radius of the ball.

Nevertheless, calculation of the relativistic angular momentum of the ball is possible with the help of elementary functions. We will illustrate this in the next section.

\section{Calculation of the Relativistic Angular Momentum of the Ball}

In the cylindrical coordinate system $r, \varphi, z$, the volume element of a fixed uniform ball is defined by the formula $d V=r d r d \varphi d z$, and the mass element of the ball is: $d m=\rho_{0} d V=\rho_{0} r d r d \varphi d z$.

The current coordinate $r$ is directed perpendicularly both to the rotation axis and to the velocity $v$. For the relativistic angular momentum of the ball's mass element we can write: $d J=\gamma_{r} r v d m$. Let us cut the ball perpendicularly to the axis $O Z$ to a number of parallel layers with thickness $d z$ and calculate the angular momentum $d J_{i}$ for one of such layers, which has a certain maximum radius $r_{i}$. Given that $v=\omega r, \gamma_{r}=\frac{1}{\sqrt{1-\omega^{2} r^{2} / c^{2}}}$, we have the following:

$d J_{i}=\int \gamma_{r} r v d m=\omega \rho_{0} d z \int_{0}^{2 \pi} d \varphi \int_{0}^{r_{i}} \frac{r^{3}}{\sqrt{1-\omega^{2} r^{2} / c^{2}}} d r=\frac{4 \pi \rho_{0} c^{4} d z}{3 \omega^{3}}\left[1-\left(1+\frac{\omega^{2} r_{i}^{2}}{2 c^{2}}\right) \sqrt{1-\omega^{2} r_{i}^{2} / c^{2}}\right]$

Now we need to integrate (4) over all the ball's layers, that is, with respect to the variable $z$. If the ball's radius is equal to $a$, we can integrate with respect to the variable $z$ from zero to $a$, that is, over one hemisphere, and then double the result in order to take into account the second hemisphere. Inside the upper hemisphere the radius $r_{i}$ of an arbitrary layer is connected with the variable $z$ by the following relation: $r_{i}^{2}=a^{2}-z^{2}$. This relation can be substituted into (4) and then integrated over all the layers:

$$
J=\frac{8 \pi \rho_{0} c^{4}}{3 \omega^{3}} \int_{0}^{a}\left[1-\left(1+\frac{\omega^{2}\left(a^{2}-z^{2}\right)}{2 c^{2}}\right) \sqrt{1-\frac{\omega^{2} a^{2}}{c^{2}}+\frac{\omega^{2} z^{2}}{c^{2}}}\right] d z
$$

The result is as follows:

$$
J=\frac{3 \pi \rho_{0} c^{4} a}{2 \omega^{3}}-\frac{\pi \rho_{0} c^{2} a^{3}}{2 \omega}-\frac{3 \pi \rho_{0} c^{5}\left(1-\frac{\omega^{2} a^{2}}{c^{2}}\right)\left(1+\frac{\omega^{2} a^{2}}{3 c^{2}}\right)}{4 \omega^{4}} \ln \frac{1+\frac{\omega a}{c}}{1-\frac{\omega a}{c}} .
$$

At low angular velocities of rotation $\omega$, we can expand the logarithm in (5) up to the terms containing the multiplier $c^{7}$ in the denominator. This gives the standard angular momentum of the ball and the first order addition:

$$
J \approx \frac{8 \pi \rho_{0} \omega a^{5}}{15}+\frac{16 \pi \rho_{0} \omega^{3} a^{7}}{105 c^{2}}=\frac{2 m \omega a^{2}}{5}+\frac{4 m \omega^{3} a^{4}}{35 c^{2}} .
$$


Another limiting case is obtained if we assume that the surface at the ball's equator moves due to rotation at the velocity reaching the speed of light. In (5) this corresponds to the fact that the ratio $\frac{\omega a}{c}$ tends to unity. If we take into account that

$$
\lim _{\omega a \rightarrow c}\left(1-\frac{\omega a}{c}\right) \ln \frac{1+\frac{\omega a}{c}}{1-\frac{\omega a}{c}}=0
$$

then in (5) the third term with logarithm disappears, and for the ball's limiting angular momentum with $\omega a=c$ we obtain:

$$
J_{\lim }=\pi \rho_{0} \omega a^{5}=\frac{3}{4} m \omega a^{2}=\frac{3}{4} m c a .
$$

Within the framework of the special theory of relativity no spherical body can reach the angular momentum equal to (7). For gravitationally bound bodies there is a softer condition for the maximum rotation velocity associated with the stability of matter at the equator, where the linear velocity has the largest value. Here the centrifugal acceleration must not exceed the acceleration from the gravitation force, which leads to the inequality: $\omega \leq \sqrt{\frac{G m}{a^{3}}}$. Substituting this angular velocity in (5) and in (6), we can estimate the maximum angular momentum of gravitationally bound bodies, knowing only their mass and radius.

In the general theory of relativity, the extremal Kerr black hole with the largest possible rotation has the angular momentum $J_{K}=\frac{G m^{2}}{c}$ and the surface radius of events $a_{K}=\frac{G m}{c^{2}}$. If we substitute $a_{K}$ in (7), we will obtain $J_{\lim }=\frac{3 G m^{2}}{4 c}<J_{K}$. Thus, in the general theory of relativity the limiting angular momentum of extremal objects increases by $25 \%$ in comparison with the result of the special theory of relativity.

\section{Calculation of the Angular Momentum of a Neutron Star and a Proton}

The results obtained are useful for estimating the angular momentum and the moment of inertia of such rapidly rotating objects as neutron stars and nucleons. The moment of inertia can be determined as the ratio of the angular momentum to the angular velocity of rotation: $I=\frac{J}{\omega}$. The mass of a uniform ball depends on the mass density and the volume of the ball: $m=\frac{4 \pi a^{3} \rho_{0}}{3}$. Substituting it into (5), we obtain the formula for the moment of inertia of a uniform ball rotating at the angular velocity $\omega$ :

$$
I=\frac{9 m c^{4}}{8 \omega^{4} a^{2}}-\frac{3 m c^{2}}{8 \omega^{2}}-\frac{9 m c^{5}\left(1-\frac{\omega^{2} a^{2}}{c^{2}}\right)\left(1+\frac{\omega^{2} a^{2}}{3 c^{2}}\right)}{16 \omega^{5} a^{3}} \ln \frac{1+\frac{\omega a}{c}}{1-\frac{\omega a}{c}} .
$$


We will use (8) to estimate the moment of inertia of the pulsar PSR J1614-2230, for which, according to [2], we know the angular velocity of rotation $\omega=1.994 \times 10^{3} \mathrm{rad} / \mathrm{s}$, the radius $a=12.8 \mathrm{~km}$, and the mass $m=1.97 M_{\odot}$, where $M_{\odot}$ is the mass of the Sun. Since for this pulsar $\frac{\omega a}{c}=8.51 \times 10^{-2}$, the surface of its equator moves at velocity of about $8.5 \%$ of the speed of light. With these data, it follows from (8) that the pulsar's moment of inertia is equal to $I=2.55 \times 10^{39} \mathrm{~kg} \cdot \mathrm{m}^{2}$, and the angular momentum reaches the value $J=5.08 \times 10^{42} \mathrm{~kg} \cdot \mathrm{m}^{2} / \mathrm{s}$. At the same time, if we would make calculation using the classical formula $J \approx \frac{2 m \omega a^{2}}{5}$, then the angular momentum would be equal to $5.11 \times 10^{41} \mathrm{~kg} \cdot \mathrm{m}^{2} / \mathrm{s}$. As we can see, in this case the relativistic formula for the angular momentum gives ten times larger value than the simple formula from classical mechanics. For a star, however, it should be taken into account that its mass density increases at the center, where it exceeds the average density approximately 1.5 times [3]. As a result, the angular momentum of the star must be less than the value $J=5.08 \times 10^{42} \mathrm{~kg} \cdot \mathrm{m}^{2} / \mathrm{s}$, calculated for a uniform ball in case of its relativistic rotation.

In quantum mechanics, it is known that the proton's spin is equal to $\frac{\hbar}{2}$, where $\hbar$ is the Dirac constant. Suppose that this value is equal to the proton's angular momentum $J_{p}$, and let us estimate how the proton rotates in this case. If we denote $\beta=\frac{\omega a_{p}}{c}$, where $a_{p}$ is the proton radius, then from (8) it follows:

$$
J_{p}=\omega I_{p}=\frac{\hbar}{2}=\frac{3 m_{p} c a_{p}}{8 \beta}\left[\frac{3}{\beta^{2}}-1-\frac{3\left(1-\beta^{2}\right)\left(1+\frac{\beta^{2}}{3}\right)}{2 \beta^{3}} \ln \frac{1+\beta}{1-\beta}\right] .
$$

In (9) the Dirac constant $\hbar$ and the proton mass $m_{p}$ are well known, and for the proton radius we will use the value $a_{p}=8.73 \times 10^{-16} \mathrm{~m}$, according to [4]. In this case (9) becomes the equation for $\beta$. The solution of this equation is the value $\beta=\frac{\omega a_{p}}{c} \approx 0.3$, from which we obtain the value of the proton's angular velocity of rotation $\omega=1 \times 10^{23} \mathrm{rad} / \mathrm{s}$. In this case the velocity of rotation of the proton's surface reaches $30 \%$ of the speed of light.

\section{Conclusion}

Using division of a ball into a number of parallel layers, we find in (5) a formula for the relativistic angular momentum of a uniform ball expressed in terms of elementary functions. According to (6), at low angular velocities of rotation $\omega$ the relativistic correction to the standard angular momentum increases in proportion to $\omega^{3}$, that is, in proportion to the cube of the rotation velocity. At sufficiently high rotation velocities the angular momentum changes as a logarithmic function. The limiting angular momentum of the ball is reached when the surface points at the equator of the ball move at the speed of light, while the angular momentum is expressed by formula (7).

Formula (5) can be used to calculate the relativistic angular momentum of a thin spherical shell with thickness $d: J_{d}=J_{a}-J_{a-d}$, where $J_{a}$ is the angular momentum of a ball with the radius $a$. In this case, a ball with reduced radius $a-d$ and angular momentum $J_{a-d}$ is embedded in this ball. 
Thus, the angular momentum $J_{d}$ of the shell becomes a function of the radius, angular velocity of rotation, mass density and thickness of this shell.

With this in mind, any rotating spherical body can be divided into a number of spherical shells, each of which has its own radius, angular velocity of rotation and mass density, and, accordingly, its own angular momentum $J_{d}$. To calculate the angular momentum of a spherical body it is only necessary to sum up the angular momenta of all the body's shells. The accuracy of the result will depend on the number of the shells used and on the accuracy of the mass density distribution and of the angular velocity of rotation inside the body.

We use the results obtained to calculate the angular momentum and the moment of inertia of the neutron star PSR J1614-2230. It turns out that the relativistic angular momentum is ten times larger than the angular momentum according to the nonrelativistic formula.

For a proton we determine the corresponding angular velocity of rotation based on its quantum spin. In this case the velocity of the proton's equatorial points reaches $30 \%$ of the speed of light. As for the star PSR J1614-2230, the velocity of its equatorial points reaches $8.5 \%$ of the speed of light. We need to add that at present the fastest rotating pulsar [5] is PSR J1748-2446ad with the angular velocity of rotation $\omega=4,498 \times 10^{3} \mathrm{rad} / \mathrm{s}$. If we assume that by mass and size it is the analogue of PSR J1614-2230, then at the assumed radius $a=12.8 \mathrm{~km}$ the relative velocity at the star's surface could reach the value $\frac{\omega a}{c} \approx 0.19$. In this case, the star could rotate at the velocity at the equator of the order of $19 \%$ of the speed of light, which is comparable to the rotation of the proton surface.

\section{References}

[1] Molina A. and Ruiz E. An approximate global solution of Einstein's equations for a differentially rotating compact body. General Relativity and Gravitation, Vol. 49, no 10, pp. 135 (2017). doi:10.1007/s10714-017-2297-5.

[2] Demorest P. B., Pennucci T., Ransom S.M., Roberts M.S.E., Hessels J.W.T. A two-solar-mass neutron star measured using Shapiro delay. Nature. Vol. 467 (7319), pp. 1081-1083 (2010). doi:10.1038/nature09466.

[3] Fedosin S.G. Estimation of the physical parameters of planets and stars in the gravitational equilibrium model. Canadian Journal of Physics, Vol. 94, no. 4, pp. 370-379 (2016). doi:10.1139/cjp-2015-0593.

[4] Fedosin S.G. The radius of the proton in the self-consistent model. Hadronic Journal, Vol. 35, no. 4, pp. 349-363 (2012). doi:10.5281/zenodo.889451.

[5] Hessels J.W.T., Ransom S.M., Stairs I.H., Freire P.C.C., Kaspi V.M., Camilo F. A Radio Pulsar Spinning at $716 \mathrm{~Hz}$. Science. Vol. 311 (5769), pp. 1901-1904 (2006). doi:10.1126/science. 1123430 . 